\title{
Percepción sobre educación virtual en estudiantes universitarios de Ciencias de la Salud, en tiempos de COVID-19
}

Perception on virtual education in university students of Health Sciences, in times of COVID-19 Percepção sobre educação virtual em estudantes universitários de Ciências da Saúde, no tempo da COVID-19

\author{
Jackelin Callasaca-Goyzueta ${ }^{\text {Ia }}$ \\ Kimberly Gutiérrez-Pantigoso ${ }^{10}$ \\ Edith Cotrado-Estalla' \\ Helbert Campos-Condori ${ }^{1 a}$ \\ Keidy Ayhuasi-Gomez ${ }^{\text {ia }}$ \\ Rubí Velarde-Quispe $e^{1 a}$ \\ Diana Huanco Apaza'
}

\section{Resumen}

Objetivo: Conocer la percepción de estudiantes universitarios de Ciencias de la Salud sobre la educación virtual. Material y métodos: Estudio descriptivo en 293 estudiantes de la Facultad de Ciencias de la Salud de la Universidad Nacional Jorge Basadre Grohmann en Tacna, Perú, durante el segundo semestre del año 2020. Se aplicó un cuestionario virtual previo consentimiento informado con 26 preguntas cerradas. Se utilizó estadística descriptiva y los datos se analizaron con STATA versión 16. Resultados: En el desarrollo de clases virtuales, los estudiantes utilizan con mayor frecuencia celular (65\%) y laptop (62,4\%), con internet inalámbrico (59,3\%). El $87,7 \%$ percibió que su enseñanza y su formación se ha visto afectada y es insuficiente, debido a la falta de adaptación del docente a esta modalidad y a la falta de recursos audiovisuales para la práctica clínica $(73,4 \%)$; consideran muy importante que las clases prácticas sean presenciales (91,1\%). Se presentaron efectos negativos en la situación socioeconómica de los alumnos (54,6\%) e incertidumbre sobre su futuro académico. Los estudiantes percibieron problemas en su salud mental principalmente estrés (30,3\%) y ansiedad (21,5\%); incluso malestares físicos como dolor de espalda (21,8\%) y ardor de ojos (21,6 \%). Conclusión: La percepción de estudiantes universitarios de https://orcid.org/0000-0001-9687-8384

Ciencias de la Salud frente a la educación virtual en torno a diferentes condiciones del aprendizaje es negativa. Sugieren retorno a clases presenciales principalmente para la práctica clínica.

Palabras clave: SARS-CoV-2, escuelas para profesionales de salud, educación a distancia, elearning, percepciones

\section{Abstract}

Objective: To know the perception of university students of health sciences about virtual education. Material and methods: Descriptive study in 293 students of the Faculty of Health Sciences of the Jorge Basadre Grohmann National University in Tacna, Peru, during the second semester of the year 2020. A virtual questionnaire with prior informed consent with 26 closed questions was applied. Descriptive statistics were used and data were analyzed with STATA version 16. Results: In the development of virtual classes, students use more cell frequency (65\%) and laptops (62.4\%), with wireless internet (59.3\%). $87.7 \%$ felt that their teaching and training has been affected and is insufficient, due to the lack of adaptation of the teacher to this modality and the lack of audiovisual resources for clinical practice (73.4\%); consider it very important that the practical classes are face-to- 
face (91.1\%). There were negative effects on the socio-economic situation of students (54.6\%) and uncertainty about their academic future. Students perceived problems in their mental health mainly stress (30.3\%) and anxiety (21.5\%); even physical discomfort such as back pain (21.8 $\%)$ and burning eyes (21.6\%). Conclusion: The perception of university students of Health Sciences versus virtual education around different learning conditions is negative. They suggest a return to face-to-face classes mainly for clinical practice.

Keywords: SARS-CoV-2, health science schools, distance learning, e-learning, perceptions

\section{Resumo}

Objetivo: Conhecer a percepção de estudantes universitários de ciências da saúde sobre a educação virtual. Material e métodos: Estudo descritivo em 293 estudantes da Faculdade de Ciências da Saúde da Universidade Nacional Jorge Basadre Grohmann em Tacna, Peru, durante o segundo semestre do ano 2020. Foi aplicado um questionário virtual com consentimento informado com 26 perguntas fechadas. Foram utilizadas estatísticas descritivas e os dados foram analisados com a versão 16 do STATA.

Resultados: No desenvolvimento de aulas virtuais, os estudantes utilizam com maior frequência celular (65 \%) e laptop (62,4\%), com internet sem fio (59,3\%). 87,7 \% consideraram que o seu ensino e a sua formação foram afectados e são insuficientes, devido à falta de adaptação do docente a esta modalidade e à falta de recursos audiovisuais para a prática clínica $(73,4 \%)$; consideram muito importante que as classes práticas sejam presenciais (91,1\%). Registaram-se efeitos negativos na situação socioeconómica dos alunos (54,6 \%) e incerteza quanto ao seu futuro académico. Os estudantes detectaram problemas de saúde mental, principalmente stress (30,3\%) e ansiedade (21,5 $\%)$, incluindo dores nas costas (21,8\%) e ardor nos olhos (21,6 \%). Conclusão: A percepção dos estudantes universitários de ciências da saúde em relação à educação virtual em torno de diferentes condições de aprendizagem é negativa. Sugerem retorno a aulas presenciais principalmente para a prática clínica.
Palavras-chave: SARS-CoV-2, escolas de ciências da saúde, educação à distância, e-learning, percepções

\section{Introducción}

A finales del 2019, en la ciudad de Wuhan-China se originó la pandemia del COVID-19.' En el Perú, se informó el primer caso el 6 de marzo del 2020, por tal motivo, el gobierno tomó medidas sanitarias como el cierre de fronteras y una cuarentena obligatoria ${ }^{2}$ que afectó los ámbitos socioeconómicos, culturales y por consiguiente el sistema educativo, quienes enfrentan nuevos retos, ${ }^{3}$ estableciendo medidas para no detener la educación.

La educación virtual ha sido definida como la educación a distancia, a través del ciberespacio, mediante la conexión y uso de internet, que no necesita un tiempo y espacio específico, dando un nuevo escenario de comunicación entre docentes y estudiantes. ${ }^{4}$

El proceso de transformación generado por la COVID-19 ha impuesto diferentes métodos de enseñanza y aprendizaje. ${ }^{5}$ En tanto, diferentes estudios han evidenciado el impacto negativo de estos métodos aplicados a la enseñanza en los estudiantes universitarios. En marzo del 2020, la UNESCO indicó que el 87 \% de la población estudiantil presenta problemáticas tales como el aislamiento social, cuestiones financieras, la conectividad a internet y dificultad para establecer horarios regulares, ${ }^{6}$ lo que generó repercusiones en la salud mental, ya sea ansiedad, estrés por sobrecarga académica, frustración y deserción universitaria. Estas repercusiones suelen ser temporales si se cuenta con los adecuados recursos tecnológicos. En caso de ser constantes en un siguiente semestre, se podría desencadenar problemas más graves en la salud mental dentro del marco de la cuarentena por COVID-19.

Esteche $^{3}$ afirma que el factor económico podría llegar a ser determinante en la deserción universitaria, debido al salario mínimo que perciben los estudiantes paraguayos con trabajo independiente. 
De igual manera, Pérezet al. ${ }^{8}$ analiza el modelo de enseñanza a distancia en España, teniendo una valoración negativa respecto a esta, por la falta de adaptación de los docentes a las circunstancias personales y académicas de los universitarios.

Es por ello que, Bao y Filius et al. ${ }^{9}$ argumentan que la enseñanza virtual exclusiva requiere una planificación e inversiones significativas de todos los sectores, pues publicar diapositivas de PowerPoint para que los estudiantes las lean no constituye enseñanza virtual. No obstante, algunos estudios plantean la eficacia de la modalidad semipresencial en la educación superior, siendo fundamental para ello la implementación de plataformas virtuales y capacitaciones para docentes y estudiantes. ${ }^{10}$

Para lo cual nos preguntamos: ¿Cuáles son las percepciones acerca de la educación virtual en tiempos de COVID-19, periodo 2020-II, en los estudiantes de la Facultad de Ciencias de la Salud (FACS) de la Universidad Nacional Jorge Basadre Grohmann?

El presente trabajo de investigación se enfocó en el análisis de la percepción de los estudiantes de FACS de la Universidad Nacional Jorge Basadre Grohmann para una mejor observación del impacto de la pandemia de la COVID-19. Centrándonos en las primeras experiencias de su educación virtual (un aspecto poco conocido en nuestra localidad) y los efectos en la calidad y el desarrollo de la enseñanza impartida en estos tiempos. Los resultados obtenidos del estudio también son de utilidad para las autoridades universitarias, quienes podrán diseñar mejores escenarios educativos a corto y mediano plazo para la preparación integral del futuro profesional de la salud buscando su óptimo desarrollo.

En base a lo expuesto, los objetivos del presente estudio son identificar percepciones acerca de los recursos de aprendizaje, factores relacionados con la metodología de enseñanza, el ámbito familiar y del medio externo en la educación virtual. Así como los efectos de la educación virtual sobre la salud psicofísica.

\section{Material y métodos}

Estudio observacional y descriptivo. De 1093 estudiantes de la Facultad de Ciencias de la Salud (FACS) de la Universidad Nacional Jorge Basadre Grohmann (UNJBG) en Tacna, matriculados en el año 2020, se incluyó a 293 estudiantes que desearon participar en el estudio, previo consentimiento informado, se excluyó aquellos con permiso por salud o por otros motivos, que hayan separado matrícula, que pertenezcan al primer año de estudios y con cuestionarios incompletos o duplicados.

La principal variable de estudio fue la percepción hacia la educación virtual, la cual tiene 4 dimensiones a medir: recursos de aprendizaje virtual, metodología de enseñanza, factores relacionados al ámbito familiar y factores externos, y efectos de la salud psicofísica. Estas dimensiones se midieron mediante preguntas que conformaron la encuesta elaborada como método para la obtención de datos a una escala nominal, que incluye alternativas con respuestas variadas, escala ordinal y de intervalo, dependiendo de la interrogante. El valor de cada dimensión se estableció según la perspectiva positiva o negativa que refieran las respuestas en cada encuesta resuelta.

La técnica que se utilizó para la obtención de datos fue por medio de una encuesta virtual. Los datos se recopilaron durante los meses de enero y febrero del año 2021, previa coordinación con los delegados representantes de cada salón de cada escuela. El instrumento fue revisado por un experto en el tema y fue difundido a través de los grupos de WhatsApp. El instrumento incluyó información sobre datos sociodemográficos y 26 preguntas, 25 cerradas y 1 abierta con un tiempo de duración aproximado de 10 minutos para responder.

La base de datos obtenida por la plataforma Google Forms se exportó al Software Excel, donde se realizó un control de la calidad de información y las codificaciones respectivas, los cuestionarios con datos faltantes e inconsistentes fueron depurados. Los datos 
fueron exportados al Software de STATA Versión 16 para su análisis. Se utilizó estadística descriptiva como frecuencias absolutas y relativas para expresar los resultados.

En los aspectos éticos se consideró la confidencialidad de los participantes, el consentimiento informado y no se reporta ningún tipo de compensación por la realización de las encuestas virtuales.

\section{Resultados}

Los hallazgos que se presentan a continuación parten del análisis del cuestionario. Se obtuvo como resultados lo siguiente:

\section{Características demográficas}

De los 303 estudiantes que accedieron a la encuesta, solo 293 (96,7\%) la respondieron en su totalidad, con un rechazo del 3,3\%, y ninguna encuesta incompleta. Los participantes se caracterizaron por ser en su mayoría mujeres (77,1\%). Los estudiantes procedían de todos los niveles de la Facultad de Ciencias de la Salud, perteneciendo principalmente al segundo año de estudios (31,4\%) y 26,3\% pertenecen a la Escuela de Obstetricia (Tabla 1).

Tabla 1

Características sociodemográficas de estudiantes universitarios de la salud. Universidad Nacional Jorge Basadre Grohmann, Tacna, 2020-II

\begin{tabular}{lcc}
\hline Característica & $\mathbf{N .}^{\circ}$ & $\%$ \\
Sexo & 67 & 22,9 \\
\hline Masculino & 226 & 77,1 \\
Femenino & & \\
\hline Carrera universitaria & 59 & 20,1 \\
\hline Medicina Humana & 45 & 15,4 \\
Enfermería & 68 & 23,2 \\
Odontología & 44 & 15 \\
Farmacia y Bioquímica & 77 & 26,3 \\
Obstetricia & & 31,4 \\
\hline Año de estudio & 92 & 23,6 \\
\hline Segundo & 69 & 20,1 \\
Tercero & 59 & 21,8 \\
Cuarto & 64 & 3,1 \\
Quinto & 9 & \\
Sexto & & \\
\hline
\end{tabular}




\section{Recursos de aprendizaje virtual}

El dispositivo más utilizado fue el celular en las carreras de Enfermería (73,3\%), Farmacia y Bioquímica (61,4\%), y Obstetricia (70,9\%), aquí hay una diferencia en comparación con Medicina Humana $(79,7 \%)$ y Odontología (69,1\%), donde el dispositivo más utilizado fue la laptop. El tipo de conexión habitual a internet es igual en toda la FACS, siendo el más usado el internet inalámbrico $(59,3 \%)$ y el $73,4 \%$ de los estudiantes de la FACS considera que la universidad nacional de Tacna no se ha adecuado a la docencia virtual ni mantiene el mismo nivel de la docencia presencial. Con respecto al tiempo de uso de dispositivos electrónicos, las carreras de Enfermería (51,1\%) y Farmacia (34,1\%) tienen un promedio de 9 a 12 horas de uso, en cambio las carreras de Medicina Humana (40,7 \%) y Odontología (38,2\%) tienen un promedio de uso de 6 a 9 horas, la carrera de Obstetricia (40,3\%) tiene en mayor promedio de uso con más de 12 horas de uso al día (Tabla 2).

Tabla 2

Recursos de aprendizaje virtual utilizados, según carrera universitaria. Universidad Nacional Jorge Basadre, Tacna, 2020-II

\begin{tabular}{|c|c|c|c|c|c|c|c|}
\hline & \multirow{2}{*}{\multicolumn{2}{|c|}{ FACS }} & \multirow{3}{*}{$\begin{array}{c}\text { Enfermería } \\
\%\end{array}$} & \multirow{3}{*}{$\begin{array}{c}\text { Farmacia y } \\
\text { Bioquímica } \\
\%\end{array}$} & \multirow{3}{*}{$\begin{array}{c}\text { Medicina } \\
\text { Humana } \\
\%\end{array}$} & \multirow{3}{*}{$\begin{array}{c}\text { Obstetricia } \\
\%\end{array}$} & \multirow{3}{*}{$\begin{array}{c}\text { Odontología } \\
\%\end{array}$} \\
\hline & & & & & & & \\
\hline & $\mathrm{n}$ & $\%$ & & & & & \\
\hline \multicolumn{8}{|c|}{ Dispositivo utilizado para el desarrollo de las clases } \\
\hline Celular & 192 & 65 & 73,3 & 61,4 & 54,2 & 70,9 & 64,7 \\
\hline Computadora & 93 & 31,5 & 44,4 & 22,7 & 30,5 & 30,4 & 30,9 \\
\hline Tablet & 8 & 2,7 & 0 & 0 & 11,9 & 1,3 & 0 \\
\hline Laptop & 184 & 62,4 & 60 & 52,3 & 79,7 & 50,6 & 69,1 \\
\hline \multicolumn{8}{|c|}{ Tipo habitual de conexión de internet (más usado) } \\
\hline Internet inalámbrico & 173 & 59,3 & 57,8 & 56,8 & 62,7 & 54,6 & 63,2 \\
\hline Plan celular pospago & 25 & 8,5 & 4,4 & 11,4 & 6,8 & 10,4 & 8,8 \\
\hline Plan celular prepago (recargas) & 28 & 9,5 & 6,7 & 9,1 & 8,5 & 14,3 & 7,4 \\
\hline \multicolumn{8}{|l|}{ Internet brindado por la } \\
\hline universidad & 50 & 17 & 31,1 & 18,2 & 8,5 & 19,5 & 11,8 \\
\hline
\end{tabular}

¿Crees que tu universidad se ha adaptado adecuadamente a la docencia virtual y que las clases mantienen el nivel de la docencia presencial?

\begin{tabular}{llllllll}
\hline Sí & 78 & 26,6 & 20 & 25 & 28,8 & 23,4 & 33,8 \\
No & 215 & 73,4 & 80 & 75 & 71,2 & 76,6 & 66,2 \\
\hline
\end{tabular}




\section{Metodología de la enseñanza}

El $87,7 \%$ de los encuestados percibe que su enseñanza y su formación como futuro profesional de la salud se ha visto afectada y deficiente en comparación con compañeros que no han experimentado la educación virtual. Evidenciando la interacción docente-estudiante sin mejoras en el 50,9\%. En el dictado de clases teóricas prevaleció en un 78,8 \% las clases expositivas con diapositivas junto a las lecturas $(8,2 \%)$. Los ejercicios y tareas grupales, con un $46,8 \%$, predominaron en las clases prácticas, seguido de los videos en clase (18,1\%). Los foros han sido en menor medida aplicados tanto en las clases teóricas (1,4\%) y prácticas (4,1\%).

El $91,1 \%$ de los alumnos opinaron que las enseñanzas presenciales de las clases prácticas son muy importantes. Por otro lado, en lo que refiere a la metodología en las evaluaciones, es decir, exámenes impartidos por los docentes, más de la mitad de los estudiantes las calificaron como regular (56,3\%), seguido de un 30,4\% que las calificaron como buenas. No obstante, se mostró la desazón de alrededor del $10 \%$, calificando como mal (5,5\%) y muy mal $(5,1 \%)$ las medidas adoptadas en las evaluaciones (Tabla 3).

Tabla 3

Perspectivas acerca de la educación virtual por la enseñanza impartida a los estudiantes de la Facultad de Ciencias de la Salud respecto a la educación presencial en la Universidad Nacional Jorge Basadre, Tacna 2020-II

\begin{tabular}{|c|c|c|c|c|c|c|}
\hline \multirow{2}{*}{$\begin{array}{l}\text { Perspectivas acerca de la educación } \\
\text { virtual respecto a la educación } \\
\text { presencial }\end{array}$} & \multicolumn{2}{|c|}{ Sí } & \multicolumn{2}{|c|}{ No } & \multicolumn{2}{|c|}{ Igual } \\
\hline & N..$^{\circ}$ & $\%$ & N..$^{\circ}$ & $\%$ & N..$^{\circ}$ & $\%$ \\
\hline \multicolumn{7}{|l|}{ ¿Crees que la enseñanza presencial es } \\
\hline $\begin{array}{l}\text { importante para el dictado de clases } \\
\text { prácticas? }\end{array}$ & 267 & 91,1 & 26 & 8,8 & - & - \\
\hline ¿Crees que la enseñanza y formación & & & & & & \\
\hline $\begin{array}{l}\text { se ha visto afectada y ha presentado } \\
\text { déficits respecto a promociones } \\
\text { anteriores? }\end{array}$ & 257 & 87,7 & 36 & 12,3 & - & - \\
\hline ¿Siente que en clases virtuales, la & & & & & & \\
\hline $\begin{array}{l}\text { interacción con el docente ha } \\
\text { mejorado, en comparación con las }\end{array}$ & 53 & 18,1 & 149 & 50,9 & 91 & 31,5 \\
\hline clases presenciales? & & & & & & \\
\hline
\end{tabular}

\section{Factores relacionados al ámbito familiar y factores externos}

El 90,4\% de los encuestados refirió que el lugar donde desarrollaron sus clases virtuales fue en el hogarfamiliar, más de la mitad (54,6\%) indicó que tuvo reducción del ingreso económico familiar importante y $82,9 \%$ percibe un ingreso económico por parte de familiares que financian sus estudios (Tabla 4). 
Tabla 4

Lugar donde se desarrollaron clases virtuales y percepción de estudiantes de salud respecto del ingreso económico familiar. Universidad Nacional Jorge Basadre, Tacna 2020-II

\begin{tabular}{lcc}
\hline Lugar de clases percepción ingreso familiar & $\mathbf{N}^{\circ}$ & $\%$ \\
\hline Lugar en que se ha desarrollado las clases virtuales & 263 & 90,4 \\
\hline Hogar familiar & 16 & 5,5 \\
Cuarto alquilado & 11 & 3,8 \\
Zona rural & 6 & 2,1 \\
Departamento & 9 \\
Otro & 9 \\
\hline
\end{tabular}

\section{Reducción del ingreso económico familiar}

\begin{tabular}{lcc}
\hline Nada & 24 & 8,2 \\
Muy poco & 26 & 8,9 \\
Poco & 82 & 28,2 \\
Mucho & 108 & 37,1 \\
Bastante & 51 & 17,5 \\
\hline
\end{tabular}

\section{Efectos en la salud psicofísica}

Las emociones provocadas por las clases virtuales, percibidas por los estudiantes participantes del estudio fueron: estrés (30,3\%), ansiedad (27,5\%), frustración (19,7\%), enojo (13,3\%), sosiego (7,1\%) y otras emociones (depresión, cansancio, incertidumbre o tristeza) (2,2\%). Se estimó que la carrera de Odontología resultó ser la que presenta mayor valoración de estrés provocado por las clases virtuales (37,9\%) (Tabla 5).

Tabla 5

Emociones generadas por clases virtuales, percibidas por estudiantes de la salud. Universidad Nacional Jorge Basadre, Tacna 2020-II

\begin{tabular}{lcccccc}
\hline \multirow{2}{*}{ Carrera universitaria } & \multicolumn{6}{c}{ ¿Qué emociones les provocan las clases virtuales? (\%) } \\
& Frustración & Estrés & Ansiedad & Enojo & Sosiego & Otras \\
\hline Medicina Humana & 19 & 32 & 25,9 & 14,3 & 6,1 & 2,7 \\
Enfermería & 15,3 & 33,9 & 29,8 & 13,7 & 5,6 & 1,6 \\
Odontología & 17 & 37,9 & 26,8 & 9,2 & 7,2 & 2 \\
Farmacia y Bioquímica & 18,6 & 36,3 & 25,7 & 12,4 & 6,2 & 0,9 \\
Obstetricia & 22 & 28,4 & 25 & 14 & 8,1 & 2,5 \\
\hline Total & $\mathbf{1 9 , 7}$ & $\mathbf{3 0 , 3}$ & $\mathbf{2 7 , 5}$ & $\mathbf{1 3 , 3}$ & $\mathbf{7 , 1}$ & $\mathbf{2 , 2}$ \\
\hline
\end{tabular}


Durante las clases virtuales, la percepción sobre la carga académica de los participantes fue valorada como bastante en $46,4 \%$, siendo los estudiantes de Obstetricia (55,8\%), Farmacia y Bioquímica (54,6\%), y Enfermería (53,3\%) los que tuvieron mayor percepción. Casi la totalidad de estudiantes (99,1\%) percibieron malestares a causa de la educación virtual, manifestaron haber tenido dolor de espalda (21,8\%), ardor de ojos (21,6\%), dolor de cabeza (19,5\%) y dolor de cuello (18,6\%) (Tabla 6).

Tabla 6

Percepción de malestares a causa de la educación virtual de estudiantes de la salud. Universidad Nacional Jorge Basadre, Tacna 2020-II

\section{¿Qué malestares has presentado debido a las clases virtuales?(\%)}

\begin{tabular}{lccccccc} 
Carrera universitaria & Ninguno & \multicolumn{2}{c}{ Ardor de Disminución de } & Dolor de & Dolor en el & Dolor de & Otros \\
& & ojos & la visión & espalda & cuello & cabeza & \\
\hline Medicina & 2,5 & 23,5 & 14 & 21,5 & 19 & 17,5 & 2 \\
Enfermería & 0 & 22,5 & 14,6 & 21,9 & 20,2 & 19,1 & 1,7 \\
Odontología & 1,2 & 21,6 & 16,3 & 21,2 & 18,4 & 19,2 & 2 \\
Farmacia & 0,6 & 21,7 & 19,3 & 21,7 & 17,5 & 19,3 & 0 \\
Obstetricia & 0,3 & 19,9 & 15,7 & 22,5 & 18,3 & 21,2 & 2 \\
\hline Total & $\mathbf{0 , 9}$ & $\mathbf{2 1 , 6}$ & $\mathbf{1 5 , 9}$ & $\mathbf{2 1 , 8}$ & $\mathbf{1 8 , 6}$ & $\mathbf{1 9 , 5}$ & $\mathbf{1 , 6}$ \\
\hline
\end{tabular}

Finalmente, los estudiantes de Ciencias de la Salud manifestaron que la modalidad de la educación debe ser semipresencial (53,2\%), siendo la carrera de Enfermería (66,7\%) seguida de la carrera de Farmacia y Bioquímica $(54,4 \%)$ y la carrera de Medicina Humana (54,2 \%) las que presentan mayor aceptación a dicha modalidad.

\section{Discusión}

Los resultados mostraron que los estudiantes de la Facultad de Ciencias de la Salud de la Universidad Nacional Jorge Basadre Grohmann de Tacna, tienen diferencias en los recursos electrónicos para el aprendizaje virtual, según el tipo de escuela profesional, donde el celular es el recurso más utilizado. Por otro lado, se evidenció el temor a una baja calidad en su formación profesional, puesto que la comunicación entre el docente-estudiante no ha mejorado, y que la metodología aplicada en clases teóricas con diapositivas, lecturas en prácticas y la sobrecarga académica, requeridas para solventar el proceso de aprendizaje no han sido del todo favorables. En consecuencia, la valoración de los estudiantes considera que la educación virtual ha desmejorado su salud psicofísica, provocando en ellos estrés, ansiedad y desmotivación para aprender. La mayor parte tiene una concepción negativa sobre la adaptación de la universidad a la educación virtual, presentando dificultades por parte del docente en el uso de la tecnología y el manejo de herramientas virtuales, e incluso la poca comprensión ante problemas de conectividad de sus estudiantes, teniendo como uno de los factores predisponentes la disminución en el ingreso económico familiar.

\section{Recursos de aprendizaje virtual}

Los resultados nos demuestran que si bien los estudiantes utilizan varios dispositivos en forma alterna para el desarrollo de sus actividades académicas y que la mayoría cuenta con internet inalámbrico, existe diferencias porcentuales en el uso de recursos electrónicos para el aprendizaje virtual según el tipo de escuela profesional, tres de las cinco carreras profesionales (Enfermería, Farmacia y Obstetricia) usan más el celular y menos internet inalámbrico; en cambio en Medicina y Odontología usan más la laptop como dispositivo educativo y más internet inalámbrico; esto se puede deber, 
probablemente a que existe diferencias económicas entre los estudiantes de las diferentes escuelas de esta universidad pública, o que exista mayor necesidad de disponer de un computador o laptop de algunas escuelas para el desarrollo de sus actividades académicas.

Pérez et al. ${ }^{8}$ encontró relación entre la mayor disponibilidad de recursos como equipamiento electrónico y de adquirir un servicio de calidad, en familias que poseen estudios superiores; esto quiere decir que, las familias universitarias hacen su mayor esfuerzo para adquirir equipos y servicio electrónico de mayor calidad, pues un servicio de mala calidad genera estrés en los estudiantes." Debido a que las familias de las universidades nacionales provienen de hogares con menores recursos y que su economía se ha visto afectada por la pandemia, los estudiantes han requerido apoyo de la universidad, quienes brindaron chips para la mayor conectividad de los estudiantes, pero este no fue suficiente para cubrir las necesidades educativas; este servicio fue utilizado más por los estudiantes de Enfermería, Obstetricia y Farmacia.

Sobre la adaptación de la universidad a la educación virtual, la mayor parte tiene una concepción negativa sobre este aspecto. Cabe señalar que esta adaptación fue de emergencia debido al contexto de la COVID-19. Para asegurar la equidad de educación virtual la universidad debe realizar una mayor inversión en tecnología, así como mejorar las estrategias de aprendizaje ya que los estudiantes hacen un esfuerzo para tener un acceso de calidad a la educación virtual pero este esfuerzo fue cada vez más difícil de sustentar por la disminución de su economía familiar.

\section{Metodología de la enseñanza}

Es indudable que la educación virtual requiere procesos que garanticen su calidad en la planificación, diseño e implementación curricular, además de un dinamismo en la enseñanza del docente, ${ }^{5}$ procesos que particularmente en nuestra universidad y en la Facultad de Ciencias de la Salud, no se ha llevado a cabo de la mejor forma, pues los estudiantes han expresado una percepción negativa al respecto y demuestran temor ante una baja calidad en su formación profesional respecto a promociones anteriores; es probable que los alumnos sobresalientes sean más críticos ante esta situación.

La interacción entre el docente y el alumno es muy importante en el proceso de enseñanza, incluso en la educación virtual, existe necesidad de los estudiantes de mantener comunicación y conexión con los docentes, la encuesta reveló que en la virtualidad la mitad de los estudiantes percibe que no hubo mejoras en el acto comunicativo docente-estudiante, si bien el estudiante es capaz de desarrollar mayor autonomía y algunas otras fortalezas, no obstante, recurre muchas veces al autoaprendizaje por no obtener mayor respuesta del docente ${ }^{13}$ quedándose con muchas dudas respecto a un tema en específico, influyendo directamente en su rendimiento y su bienestar emocional.

Tanto para el desarrollo de las clases teóricas virtuales y presenciales, el método comúnmente aplicado para la enseñanza fueron las clases expositivas con diapositivas y otras presentaciones multimedia ${ }^{8}$ de esta forma, se deduce que la educación virtual requiere de estrategias adicionales para su eficaz desarrollo y no solo puede valerse de diapositivas expuestas tal como se realiza presencialmente.

En Ciencias de la Salud para llevar a cabo las clases prácticas es de suma importancia el ejercicio en laboratorios, centros de salud y hospitales. En universidades como Northampton u Oxford, en el Reino Unido, se aplica el uso de maniquís como simuladores y la aplicación de sistemas de realidad virtual, en un entorno tridimensional inmersivo e interactivo (3D), donde los estudiantes son beneficiados al realizar exámenes físicos de rutina y prácticas de intervenciones quirúrgicas mientras son monitoreados a distancia por los docentes. ${ }^{14}$ En nuestra Universidad, al igual que en las demás universidades nacionales del Perú, no han podido adquirir simuladores como estos u otros materiales imprescindibles para la formación académica virtual.

Una investigación en estudiantes de la Pontificia Universidad Católica del Perú reveló que los docentes usan mayoritariamente los foros y 
lecturas, permitiendo al estudiante resolverlas en un periodo corto donde muchos de ellos no llegan a concluir el trabajo. ' Sin embargo, los docentes de la Universidad Nacional de Tacna hacen uso mínimo de foros, pero mayor empleo de lecturas sobre todo para las clases prácticas, lo cual no parece ser lo adecuado.

Cabe mencionar que, las clases prácticas presenciales ya presentaban problemas por falta de disponibilidad de los laboratorios y falta de implementación de los existentes, pero ya en un contexto virtual la situación se ha agravado y no se ha tomado a tiempo las medidas correctivas.

En ambos casos, en clases teóricas y prácticas, la situación ha sido desfavorable, en la medida que el conocimiento del estudiante no se ha reforzado, solo se generó mayor estrés y desmotivación para aprender. Todo ello a causa de dificultades presentadas por el docente para el uso de la tecnología y el manejo de herramientas virtuales, así como la falta de dinamismo con sus estudiantes para estimularlos a seguir aprendiendo.

\section{Factores relacionados al ámbito familiar y factores externos}

Los resultados muestran que casi la mayoría del total de los estudiantes desarrollan sus clases virtuales en un ambiente familiar, si bien en la mayoría de los casos los estudiantes han tenido un ambiente donde recibían sus clases virtuales no han podido controlar las interferencias familiares y los ruidos externos principalmente de la calle que en algunas ocasiones han perturbado la atención en las clases.

Si bien, el 82,9\% de los estudiantes depende del ingreso económico de sus familias, en nuestro estudio se encontró que un 54,6 \% percibió una reducción del ingreso económico, este porcentaje fue menor con respecto al pronunciamiento de la UNESCO que manifestó que un $87 \%$ de la población estudiantil presenta problemas en cuestiones financieras. Esta reducción de ingreso económico se debió al confinamiento que hubo como una de las medidas del gobierno para evitar la propagación y controlar la pandemia, durante esta etapa se cerraron empresas, tiendas, locales comerciales, restaurantes, gimnasios, cines, etc., lo que provocó un despido masivo de trabajadores. Esto ocasionó que las familias de la mayoría de los estudiantes universitarios tengan problemas en cubrir gastos del hogar, y dentro de la cual está la conectividad del internet que es uno de los factores que influyen en la educación virtual.

El factor económico en tiempos de COVID-19 afectó considerablemente la educación virtual de los estudiantes universitarios de la salud. Esto lo confirma Esteche ${ }^{3}$ en su estudio, con respecto a que el factor económico es la variable más influyente en la modalidad virtual, y debido a esto podría llegar a ser determinante en la deserción universitaria, debido al salario mínimo que perciben los estudiantes con trabajo independiente o incluso los dependientes de ingresos familiares.

\section{Efectos en la salud psicofísica}

Los estudiantes universitarios consideran que la educación virtual afectó su salud psicofísica, provocando en ellos una variedad de emociones. Antes de llevar a cabo el proceso educativo $\mathrm{Gil}^{15}$ menciona: "Los estudiantes creen que la pandemia del COVID-19 va tener un impacto negativo en ellos", poco después se demostró en la práctica un aumento de ansiedad, agobio e incertidumbre en los estudiantes universitarios. ${ }^{16}$ En nuestro estudio, los estudiantes percibieron como emoción imperante el estrés (30,2 \%) y ansiedad (27,5\%) a causa del proceso de adaptación al nuevo modelo de enseñanza. Si bien estas emociones ya se presentaban antes del estado de emergencia, los estudiantes percibieron que se incrementaron debido a diferentes circunstancias de la pandemia y de la educación virtual.

Una posible explicación, según la percepción de los estudiantes, fue la sobrecarga académica debido al incremento de actividades establecidas por los docentes, requeridas para el proceso de aprendizaje de los estudiantes. Cabe recordar que los estudiantes como los docentes se han visto obligados a adaptarse a un nuevo modelo formativo cuyos contenidos estaban diseñados para la presencialidad. Como manifiesta Salinas, ${ }^{17}$ para la formación de estudiantes en modalidad virtual, se requiere 
flexibilidad por parte de los docentes. Además, la virtualidad les exige un mejor manejo del tiempo y, por tanto, mayor disciplina y organización. Hay que añadir a esto la carencia de experiencia en el manejo de las clases virtuales por parte de algunos docentes, habilidades diferentes a las que se requerían en la enseñanza presencial.

Otro aspecto fueron los horarios inestables, pues la modalidad virtual durante una cuarentena suele vincularse con un número de horas de clase indefinido, pues como los estudiantes permanecían en casa, no había excusas para realizar clases en diferentes horarios.

Otro factor importante, fue la situación de confinamiento y la sensación de poder enfermar durante la pandemia, que ha ocasionado sobre todo mayor estrés y ansiedad en la población en general e incluso en estudiantes. Apaza ${ }^{18}$ encontró en su estudio una predisposición de malestares relacionados a reacciones psicológicas por el contexto de pandemia.

Uno de los resultados de esta falta de adaptación a la educación virtual, podría ser la presencia de malestares físicos y psicológicos que aquejan al estudiante, y probablemente también al docente, pues se sabe que la mayoría de docentes laboran activamente en el sector sanitario, en primera línea durante la pandemia. Los malestares que han presentado la mayoría de los estudiantes van desde dolores de espalda y cuello, además de ardor de los ojos, debido a la elevada cantidad de horas sentado frente al computador. Estos malestares y cambios físicos probablemente están creciendo, debido a la extensión del periodo de pandemia, falta mayores estudios.

\section{Limitaciones y fortalezas}

El presente estudio tuvo ciertas limitaciones en su desarrollo. Al realizarse una encuesta de manera virtual, esta pudo no llegar a ciertos estudiantes de salud, a pesar de ello, este es el primer reporte de las percepciones de estudiantes de la salud sobre la educación virtual en tiempos de COVID-19, en la ciudad de Tacna, que evidencia una intención de volver a la educación presencial, para realizar las prácticas, pero bajo condiciones adecuadas. Además, permite conocer los considerables efectos sobre la salud e incertidumbre que viven actualmente sobre su futuro académico.

\section{Conclusiones}

En conclusión, las percepciones acerca de los recursos de aprendizaje, indican que no todas las carreras de Ciencias de la Salud tienen los mismos recursos, que la Universidad Nacional Jorge Basadre Grohmann no brinda suficiente apoyo tecnológico, debido a que la mayoría utiliza internet inalámbrico, a pesar de los esfuerzos realizados la Universidad no ha logrado una adaptación al entorno virtual, evidenciándose mediante la exposición de los estudiantes frente a una pantalla dedicados al estudio incluso por más de 12 horas, teniendo repercusiones negativas en su salud.

En cuanto a los factores relacionados con la metodología de enseñanza de los docentes, se concluye que el $87,7 \%$ de los encuestados perciben negativamente su formación académica debido a dos causas principales, la falta de dinamismo para el desarrollo de sus clases y una ineficaz comunicación con los mismos, que los llevó a desconocer las dificultades que atravesaban los estudiantes.

Por otro lado, respecto al ámbito familiar y del medio externo en la educación virtual, se percibió que la mayoría de los estudiantes (54,6 $\%)$ perciben una reducción del ingreso económico, de la cual la mayoría es dependiente de su familia; por lo tanto, este factor ha sido significativo para la educación virtual de los estudiantes de la salud en tiempos de pandemia, además que a partir de este dependen otros factores.

Finalmente, los efectos negativos de la educación virtual sobre la salud psicofísica, se encontró que entre las emociones percibidas por los estudiantes universitarios estuvieron el estrés y ansiedad, y malestares como dolor de espalda y cuello y ardor en los ojos, a raíz de la modalidad de educación virtual que complican aún más su situación académica. 
A partir del estudio, así como de la experiencia personal de los estudiantes de Ciencias de la Salud de la Universidad Nacional Jorge Basadre Grohmann, realizamos algunas propuestas generales y concretas de actuación:

Mejorar el método de enseñanza, con el objetivo que sea más interactiva y didáctica, como videos explicativos sobre todo para las clases prácticas que es donde más deficiencias se muestran.

Ser más empáticos y comprensivos con los problemas de conectividad, dar tiempo coherente para resolver las evaluaciones parciales y disminuir la carga académica, si bien es cierto no se ocupa tiempo en movilidad, al permanecer en casa las labores del hogar y deberes también han ido en aumento para muchos estudiantes.

Respetar los horarios y aplicar pausas activas en clases, debido al estrés que ocasiona el tener horarios inestables que demanda al estudiante estar pendiente a sus redes sociales y genera cansancio mental debido a la exposición prolongada a una laptop o computador.

Futuras líneas de investigación podrían ampliar el alcance del estudio y abordar, desde la perspectiva del profesorado, el modelo de enseñanza a distancia que han implementado durante la suspensión de las clases presenciales e identificar las dificultades que han enfrentado unos y las buenas prácticas de otros. El empeño de este artículo no es otro que presentar mediante evidencias empíricas un diagnóstico con luces y sombras sobre la enseñanza a distancia impartida durante el confinamiento que sirva a las universidades para afrontar los retos que sigue deparando la COVID-19.

\section{Declaración de conflictos de interés}

Los autores declaran no tener conflicto de intereses para la ejecución del presente trabajo de investigación.

1. Cronología de la pandemia del coronavirus y la actuación de la Organización Mundial de la Salud [Internet]. Noticias ONU. 2020 [citado 4 de marzo de 2021]. Disponible en: https://news.un.org/es/story/2020/04/1472 862

2. Albitres-Flores L, Pisfil-Farroñay $Y$, GuillenMacedo K, Niño R, Alarcon-RuizC. Percepción de los internos de medicina sobre el internado durante la pandemia por COVID19 en Perú. 2020.

3. Cabaña E, Wasmuth G. Factores que inciden en la educación virtual en tiempos de pandemia (COVID-19) de los estudiantes universitarios de una universidad privada.:15.

4. Expósito CD, Marsollier RG. Virtualidad y educación en tiempos de COVID-19. Un estudio empírico en Argentina. EDUCACIÓN [Internet]. 24 de agosto de 2020 [citado 22 de noviembre de 2020];22(39). Disponible en: http://revistas.unisimon.edu.co/index.php/e ducacion/article/view/4214

5. Universidad del Atlántico, Colombia, OjedaBeltrán A, Ortega-ÁlvarezDD, Universidad del Atlántico, Colombia, Boom-Marcano EA, Universidad de La Guajira, Colombia. Análisis de la percepción de estudiantes presenciales acerca de clases virtuales como respuesta a la crisis del COVID-19. Espacios [Internet]. 5 de noviembre de 2020 [citado 22 de noviembre de 2020];41(42). Disponible en: https://revistaespacios.com/a20v41n42/a20 v41n42p07.pdf

6. Report «COVID-19 y educación superior: De los efectos inmediatos al día después. Análisis de impactos, respuestas políticas y recomendaciones» [Internet]. Guni Network. 2020 [citado 4 de marzo de 2021]. Disponible e

http://www.guninetwork.org/publication/r eport-covid-19-y-educacion-superior-delos-efectos-inmediatos-al-dia-despuesanalisis

7. Cueva MAL, Terrones SAC. Repercusiones de las clases virtuales en los estudiantes universitarios en el contexto de la cuarentena por COVID- 19: El caso de la PUCP. Propósitos y Representaciones. 2020; 8:16.

8. LópezEP, Atochero AV, Rivero SC. Educación a 
distancia en tiempos de COVID-19: Análisis desde la perspectiva de los estudiantes universitarios. RIED Revista Iberoamericana de Educación a Distancia. 2 de enero de 2021;24(1):331-50.

9. Demuyakor J. coronavirus-covid-19-andonline-learning-in-higher-institutions-ofeducation-a-survey-of-the-perceptions-ofghanaian-international-students-in-china. Online Journal of Communication and Media Technologies. 19 de mayo de 2020;10: e202018.

10. Aguilar-Salinas WE, Fuentes-Lara M de las, Justo-López A, Rivera-Castellón RE. Percepción de los Estudiantes acerca de la Modalidad Semipresencial en la Enseñanza de las Ciencias Básicas de la Ingeniería. Un Estudio de Caso Universitario. Form Univ. junio de 2019;12(3):15-26.

11. Jaap A, Dewar A, Duncan C, Fairhurst K, Hope D, Kluth D. Effect of remote online exam delivery on student experience and performance in applied knowledge tests. BMC Med Educ. 2 mde febrero de 2021;21(1):86.

12. Loda T, Löffler T, Erschens R, Zipfel S, Herrmann-Werner A. Medical education in times of COVID-19: German students' expectations - A cross-sectional study. PLoS One [Internet]. 18 de noviembre de 2020 [citado 9 de diciembre de 2020];15(11). D i s p o n i b l e e $n$ : https://www.ncbi.nlm.nih.gov/pmc/articles /PMC7673791/

13. Valero-Cedeño NJ, Castillo-Matute AL, Rodríguez-Pincay $R$, Padilla-Hidalgo $M$, Cabrera-Hernández M. Retos de la educación virtual en el proceso enseñanza aprendizaje durante la pandemia de COVID-19. Dominio de las Ciencias. 30 de octubre de 2020;6(4):1201-20.
14. Cayo-Rojas CF, Agramonte-Rosell R de la C, Cayo-Rojas CF, Agramonte-Rosell R de la C. Desafíos de la educación virtual en Odontología en tiempos de pandemia COVID-19. Revista Cubana de Estomatología [Internet]. septiembre de 2020 [citado 6 de diciembre de 2020];57(3). Disponible en: http://scielo.sld.cu/scielo.php?script=sci_ab $\mathrm{s} t \mathrm{r}$ a c t \& p i $d=S 0034$ $75072020000300017 \&$ lng=es\&nrm=iso\&tln $\mathrm{g}=\mathrm{es}$

15. Villa FG, Litago JDU, Fernández AS. Percepciones y expectativas en el alumnado universitario a partir de la adaptación a la enseñanza no presencial motivada por la pandemia de COVID-19. Revista Latina. 30 de octubre de 2020;(78):65-85

16. Cabero J, Llorente M del C, Puentes A. La satisfacción de los estudiantes en red en la formación semipresencial. comunicar. 1 de octubre de 2010;18(35):149-57.

17. Salinas, J. Entornos virtuales y formación flexible. Revista Tecnología En Marcha. [Internet]. 2004 [citado 27 de marzo de 2021]; $17(3), 87-99$. D i s p o n i ble en:https://revistas.tec.ac.cr/index.php/tec marcha/article/view/1446

18. Apaza P. CM, Seminario Sanz RS, Santa-Cruz Arévalo JE. Factores psicosociales durante el confinamiento por el Covid-19 - Perú. REV VENEZ GERENC [Internet]. 5 de junio de 2020 [citado 27 de marzo de 2021];25(90):402-13. D i s p o n i b | e e $n$ : https://www.produccioncientificaluz.org/in dex.php/rvg/article/view/32385

\section{Correspondencia:}

jrcallasaca@unjbg.edu.pe

Fecha de recepción: 11/10/2021

Fecha deaceptación: 1/12/2021 